\title{
The Effects of Paternal Perinatal Depressive and Anxiety Symptoms on Newborn Low Birth Weight
}

\author{
Sakinah Md. Rifin ${ }^{1}$ \\ ${ }^{1}$ Department of Family Medicine, Kulliyyah of Medicine, International Islamic \\ University Malaysia, Pahang, Malaysia
}

Presenter: Sakinah Md Rifin

Introduction: Despite significant improvement in health status in Malaysia, the prevalence of low birth weight (LBW) in newborns remained unchanged over the past 12 years. In the past decade, there have been assumptions regarding the potential etiologic association of psychosocial factors with LBW. It is widely believed that only mothers are affected by depression and anxiety during pregnancy. However, this study aimed to investigate the independent effects of paternal antepartum depressive and anxiety symptoms on LBW newborns in Kuantan, Pahang. Materials and Methods: A prospective cohort study of 450 expectant fathers was carried out in six conveniently selected government health clinics in Kuantan, Pahang. Paternal antepartum depressive symptoms were assessed using Edinburgh Postpartum Depression Scale (EPDS) and anxiety symptoms using Depression Anxiety Stress Scale-21 (DASS-21) and they were followed-up until delivery. Newborn birth weight of singleton live babies delivered at term ( $\geq 37$ weeks) were recorded to the nearest $0.1 \mathrm{~kg}$ from Infant and Child Health Record. Descriptive analysis along with chi-square and independent sample t-test were carried out together with multiple logistic regression model to identify predictors of LBW. Results: After adjusting for potential cofounders, paternal depressive $(\mathrm{OR}=4.29 ; 95 \% \mathrm{Cl} 1.17-15.72)$ and paternal anxiety $(\mathrm{OR}=4.62 ; 95 \% \mathrm{Cl}$ 1.74-12.26) symptoms were significantly associated with LBW $(\leq 2.5 \mathrm{~kg})$. Conclusion: This study showed that paternal depressive and anxiety symptoms increased the risk of newborn LBW. Therefore, it is crucial to detect and manage the depressive and anxiety symptoms for expectant fathers during antenatal care to reduce the burden of LBW. 